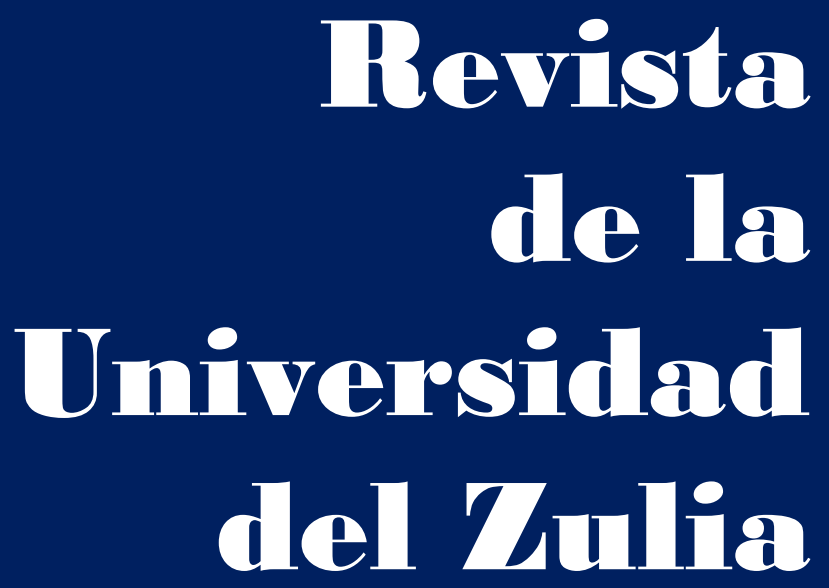

Fundada en 1947

por el Dr. Jesús Enrique Lossada

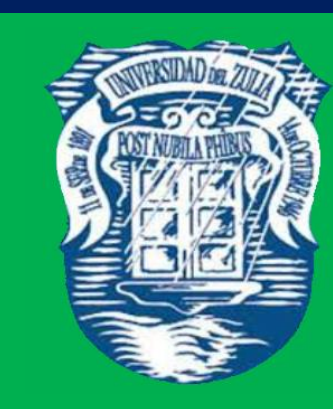

Ciencias del

Agrad,

Ingemiería

y Tecinología

\section{Aกัต 13 No $\mathbf{3 6}$} Enero - Abril 2022

Tercera Épaca

Maracailbo-Venezuela 


\title{
Gestión del Laboratorio Bioambiental de la Universidad Nacional Experimental del Táchira (UNET) como referente de datos físico- químicos de los suelos del estado Táchira-Venezuela
}

\author{
Luimar Álvarez* \\ Cristopher Camargo** \\ Alexandro Barbosa*** \\ Juan Montilla $* * * *$ \\ Erika Trujillo***** \\ Lency Chacón ${ }^{* * * * *}$
}

RESUMEN

Los laboratorios de suelos se encargan de ofrecer servicio al público para el análisis de suelos, con un estricto control de calidad que permita la confiabilidad de sus resultados. La correcta interpretación de sus parámetros permite realizar recomendaciones acertadas en beneficio del productor con criterios de sostenibilidad. El objetivo de este artículo es destacar la gestión del Laboratorio Bioambiental de la Universidad Nacional Experimental del Táchira (UNET), como centro de análisis de muestras de suelos y referencia de datos físico-químicos derivados de los mismos para el estado Táchira-Venezuela, mostrando su cobertura geográfica y los promedios generales. Para ello se ubicaron las muestras registradas mediante la utilización de un sistema de información geográfica (SIG), asegurando contener datos de 29 municipios, y se analizaron de forma general para el estado Táchira, entre los años 2015-2019. Los resultados mostraron que la mayoría de los suelos son de textura gruesa y los valores físico-químicos indican que el estado Táchira cuenta con tierras aptas para la actividad agrícola y ganadera.

PALABRAS CLAVE: suelo; Sistema de Información Geográfica; fertilidad del suelo; procesamiento de datos.

*Universidad Nacional Experimental del Táchira. Decanato de Extensión. Departamento de Ingenieria Ambiental, Decanato de Extensión. Laboratorio Bioambiental UNET / Decanato de Investigación. Grupo de Investigación en Biotecnología Agrícola y Ambiental. San Cristóbal, Venezuela. ORCID: https://orcid.org/0000-0001-5641-4331. E-mail: luimaralvarez@gmail.com

**Universidad de Los Andes. Facultad de Ciencias Forestales y Ambientales, Departamento de Ordenación de Cuencas Hidrográficas. Mérida, Venezuela. ORCID: https:/orcid.org/0000-0003-1867-4591. E-mail: ccamargoroa@gmail.com

***Universidad Nacional Experimental del Táchira. Departamento de Agronomía, Decanato de Extensión. Laboratorio Bioambiental UNET / Decanato de Investigación. Grupo de Investigación en Biotecnología Agrícola y Ambiental. San Cristóbal, Venezuela. ORCID: https://orcid.org/0000-0002-5115-6043. E-mail: abarbosa033@gmail.com

****Universidad Nacional Experimental del Táchira. Decanato de Extensión/ Departamento de Ingeniería en Producción Animal. San Cristóbal, Venezuela. ORCID: https://orcid.org/0000-0002-7147-388. E-mail: jcmontillav@gmail.com

*****Universidad Nacional Experimental del Táchira. Decanato de Extensión. Coordinación de Extensión Agraria. San Cristóbal, Venezuela. ORCID: https://orcid.org/0000-0002-4541-1255. E-mail: erikaunet@gmail.com

******Universidad Nacional Experimental del Táchira. Decanato de Extensión. Laboratorio Bioambiental UNET. San Cristóbal, Venezuela. ORCID: https://orcid.org/0000-0003-1990-1282. E-mail: lency6@gmail.com

Recibido: 01/11/2021

Aceptado: 17/12/2021 


\section{Management of the Bioenvironmental Laboratory of the National Experimental University of Táchira (NEUT) as a reference of physical-chemical data of the soils of Táchira state-Venezuela}

ABSTRACT

Soil laboratories are responsible of offering a service to the public for the analysis of soils, with a strict quality control that allows the reliability of their results. The correct interpretation of its parameters allows to make accurate recommendations for the benefit of the producer with sustainability criteria. The objective of this article is to distinguish the management of the Bioenvironmental Laboratory of the National Experimental University of Tachira (NEUT), as a center for the analysis of soil samples and reference of physicochemical data derived from them for the Táchira state-Venezuela, showing its geographic coverage and general averages. For this purpose, the registered samples were located through the use of a geographic information system (GIS), ensuring they contain data from 29 municipalities, and they were analyzed in a general way for the state of Táchira, between the years 2015-2019. The results showed that most of the soils have a coarse texture and the physical-chemical values indicate that the state of Táchira has suitable lands for agricultural and cattle activities.

KEY WORDS: soils; Geographic Information System; Soil fertility; data processing.

\section{Introducción}

A nivel mundial los laboratorios de suelos cuentan con una gran responsabilidad al ofrecer servicios de análisis y recomendaciones de fertilización, los cuales serán más exactos, precisos y adecuados en la medida en que el muestreo de suelos haya sido representativo del lote de interés, es decir, recolectando un mayor número de muestras compuestas (Roberts y Henry, 2001; Lizcano et al., 2017). El análisis de suelos es una herramienta que reúne una serie de variables analizadas de forma integral por un Ingeniero agrónomo o un asistente técnico experto, el cual permite dar una interpretación para un plan de fertilización de un determinado cultivo; y es por esta razón que es importante realizar de modo correcto el muestreo agrícola para evitar una recomendación equivocada que ocasione una baja productividad al agricultor (Lizcano et al., 2017). Además, los laboratorios de análisis de fertilidad de los suelos permiten 
generar mapas digitales y georreferenciados con el fin de planificar un mejor uso del suelo e incrementar el rendimiento de los cultivos (Villareal, 2018), y también sirven como soporte fundamental para el desarrollo de investigaciones relacionadas con el área agrícola gracias a la determinación de los parámetros físico-químicos que ofrecen (Blanco et al., 2021).

A pesar de no contar con datos actualizados de los suelos del estado Táchira, se puede mencionar de modo general, con respecto al uso y disponibilidad de tierras, que un 22,4\% (198 001 ha) de estas han sido destinadas unicamente a la agricultura (MARNR, 1986; Valero, 2009); De igual modo se conoce que la actividad económica predominante en municipios como: Jáuregui, José María Vargas, Michelena, Lobatera, Andrés Bello, Seboruco y Ayacucho, entre otros, es precisamente la actividad agrícola (Caraballo, 2003; Castillo et al., 2014). Por ello, contar con análisis de suelos, constituye la base para obtener el mayor provecho de este recurso en el estado.

El Laboratorio Bioambiental de la Universidad Nacional Experimental del Táchira (UNET), anteriormente conocido como el Laboratorio de Suelos y Agua, desde sus comienzos en marzo de 1983, ha prestado servicio al público, especialmente a los productores agrícolas de la región, ofreciéndoles análisis de rutina de muestras superficiales de suelos, mediante los cuales los productores pueden conocer el recurso que están utilizando, hallar sus limitaciones y potencialidades, así como obtener de personal especializado en el área, las recomendaciones agrícolas óptimas que mejoren la relación costo/beneficio con un enfoque ambientalmente aceptable (Martínez et al., 2006). Hoy día el Laboratorio también ofrece servicios de análisis de agua y de alimentos y ha ampliado su gestión en los últimos años a ofrecer servicios y asesorías especializadas en cualquier área del sector agrícola al contar con personal altamente calificado que forma parte de la UNET.

El Laboratorio Bioambiental cuenta con una considerable y variada información sobre características físicas y químicas de los suelos del estado Táchira, la cual permitó a Martínez et al. (2006), mostrar a partir del empleo de un Sistema de Información Geográfica (SIG), como la misma se puede organizar, analizar y divulgar. No obstante, en el Laboratorio es común que luego de entregar los resultados de los análisis de rutina al agricultor, los datos queden represados como información interna sin ser procesados con la minuciosidad requerida para 
fines de interés académico, subutilizandose así una información confiable, de calidad y referencial para el estado Táchira.

En vista de esta situación, el objetivo de este trabajo es destacar la gestión del Laboratorio Bioambiental UNET como centro de análisis de muestras de suelo y referente de datos físico-químicos de los suelos del estado Táchira, mostrando la ubicación geográfica a partir de la cual provienen los datos y los valores promedios encontrados en los análisis de rutina.

\section{Metodología}

\subsection{Manejo de las muestras en el Laboratorio}

Las muestras de suelo cuentan con un manejo desde la toma de las mismas hasta la entrega de resultados al cliente. Previa consulta, el cliente se asesora de la forma correcta de la toma de muestra y traslado de la misma al Laboratorio (Figura 1). Seguidamente se realizan los análisis de rutina que generalmente son: Textura (Bouyoucus), Materia orgánica (Walkley y Black), Fósforo disponible (Bray I 0,03 M NH4F + 0,025 M HCl), Cationes intercambiables (extracción con acetato de amonio $1 \mathrm{M} \mathrm{pH} \mathrm{7,0),} \mathrm{pH} \mathrm{(pH-metro)} \mathrm{y} \mathrm{Conductividad} \mathrm{eléctrica}$ (conductímetro).

\subsection{Metadatos y ubicación de las muestras}

Con la finalidad de ubicar puntualmente y de forma fidedigna las muestras recibidas en el Laboratorio Bioambiental UNET, se decidió procesar los datos de los últimos cinco años laborales ininterrumpidos, las cuales corresponden a las muestras de suelos sometidas a análisis durante los años 2015 y 2019 para el estado Táchira (no se incluyeron los años siguientes debido a las diversas interrupciones de actividades del Laboratorio ocasionada por el COVID-19). Los mismos fueron localizados mediante el software de sistema de información geográfica QGis versión 3.16.13, a partir de la información suministrada en la planilla de recepción de análisis (Figura 2), la cual fue precisada con capas vectoriales políticoadministrativas del SIGOT (Sistema de Información para la Gestión y Ordenación del Territorio) y un mosaico ráster de cartas a escala l: 100000 desarrolladas por el IGVSB (Instituto Geográfico Venezolano Simón Bolívar), aunado a aplicaciones web como Google 
Earth (https://earth.google.com/web/), Google Map (https://www.google.com/maps/) y el Directorio de Los Gelvez (https://gelvez.com.ve/), entre otras.
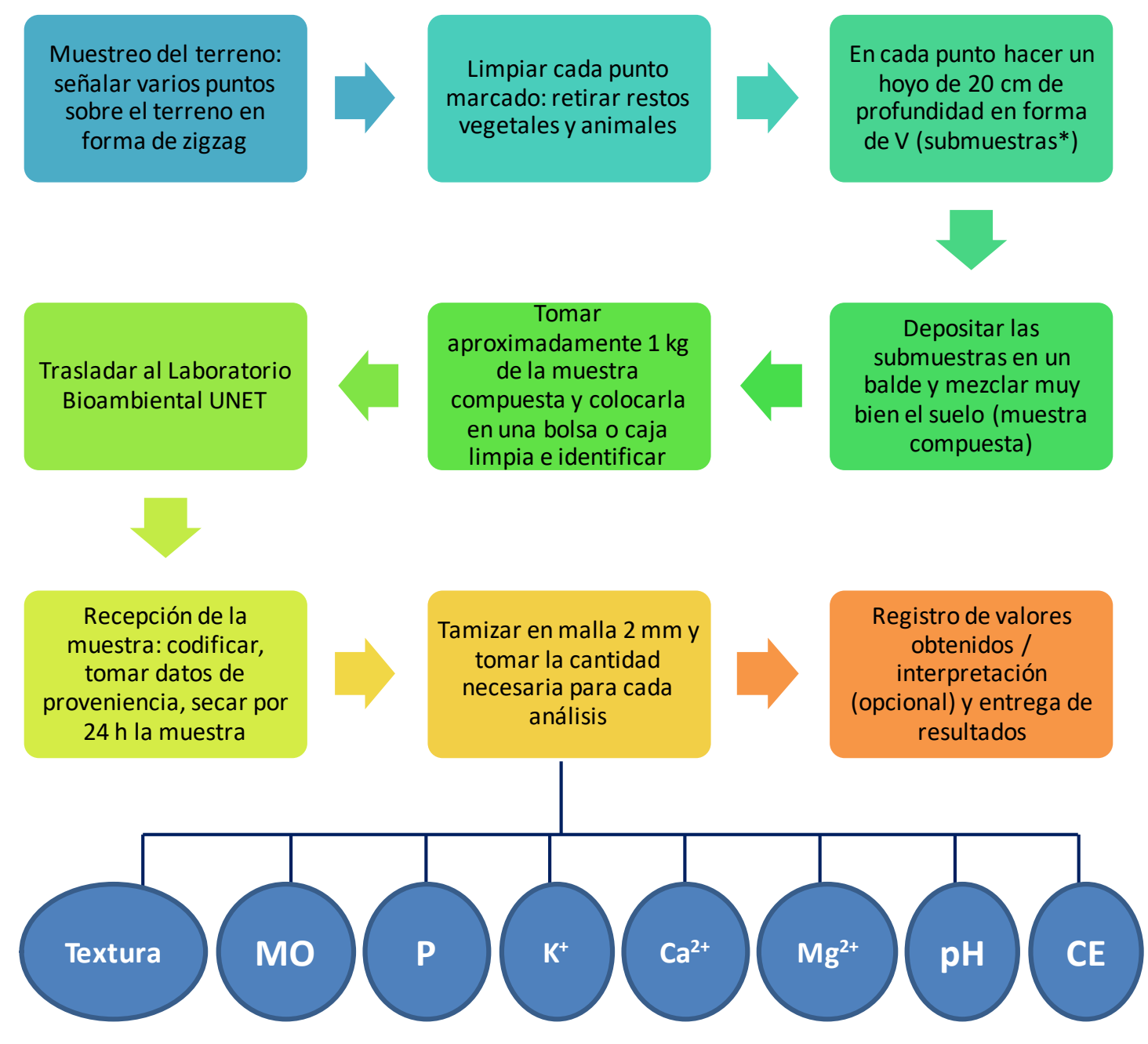

Figura 1. Flujograma del procesamiento de las muestras del suelo en el Laboratorio Bioambiental UNET. $\mathrm{MO}$ = materia orgánica; $\mathrm{CE}$ = conductividad eléctrica. ${ }^{*} \mathrm{El}$ número de submuestras se determina de acuerdo a las características del lote de terreno con previa consulta al Laboratorio.

\subsection{Análisis de datos}

Los datos de cada una de las propiedades de los suelos se procesaron con Microsoft Office Excel 2007. Asimismo, los resultados de los ánalisis de los suelos se interpretaron bajo los criterios establecidos por PALMAVEN (1992). Los datos de la propiedades de los suelos se analizaron con estadísica descriptiva (media, desviación estandár, valor mínimo y máximo). Además, se realizó el análisis de correlación de Spearman, un método estadístico no 
REVISTA DE LA UNIVERSIDAD DEL ZULIA. 3ㅜépoca. Año 13 N $^{\circ}$ 36, 2022 Luimar Álvarez et al. /// Gestión del Laboratorio Bioambiental de la Universidad Nacional Experimental... 173-188 DOI: http://dx.doi.org/10.46925//rdluz.36.12

paramétrico, que pretende examinar la intensidad de asociación entre dos variables cuantitativas (Mondragón, 2014) derivada de un análisis matricial con propiedades del algera lineal (Restrepo et al., 2007), utilizando el software Past3 (definiendo como variables cada una de las propiedades estudiadas). Para ello se estableció una prueba de hipótesis a un intervalo de significancia del 95\%, estableciéndose un valor p asociado al estadístico de contraste menor que alfa $(0,05)$ para rechazar como hipótesis nula $(\mathrm{HO}=0)$, que las variables presentan una asociación entre sí $(\mathrm{Hl} \neq 0)$.
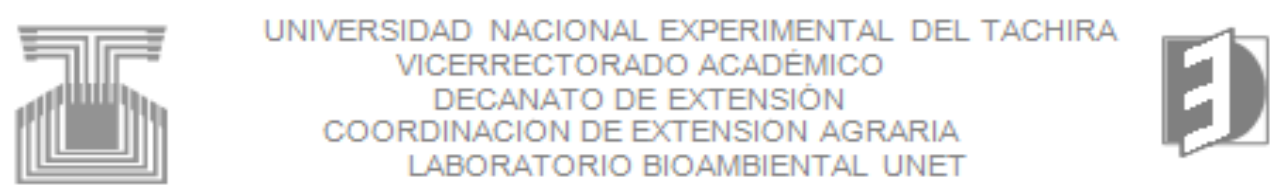

\section{RECEPCIÓN DE SUELOS PARA ANÁLISIS}

FECHA DE RECEPCIÓN: FECHA DE ENTREGA:

\section{INFORMACIÓN DEL CLIENTE}

Nombre del Cliente:

Finca o Empresa:

Dirección/Localidad:

Ciudad:

\section{ad:}

Municipio/Parroquia
Cédula - Rif

Teléfono:

Email:

Estado:

\section{INFORMACIÓN DE LA MUESTRA}

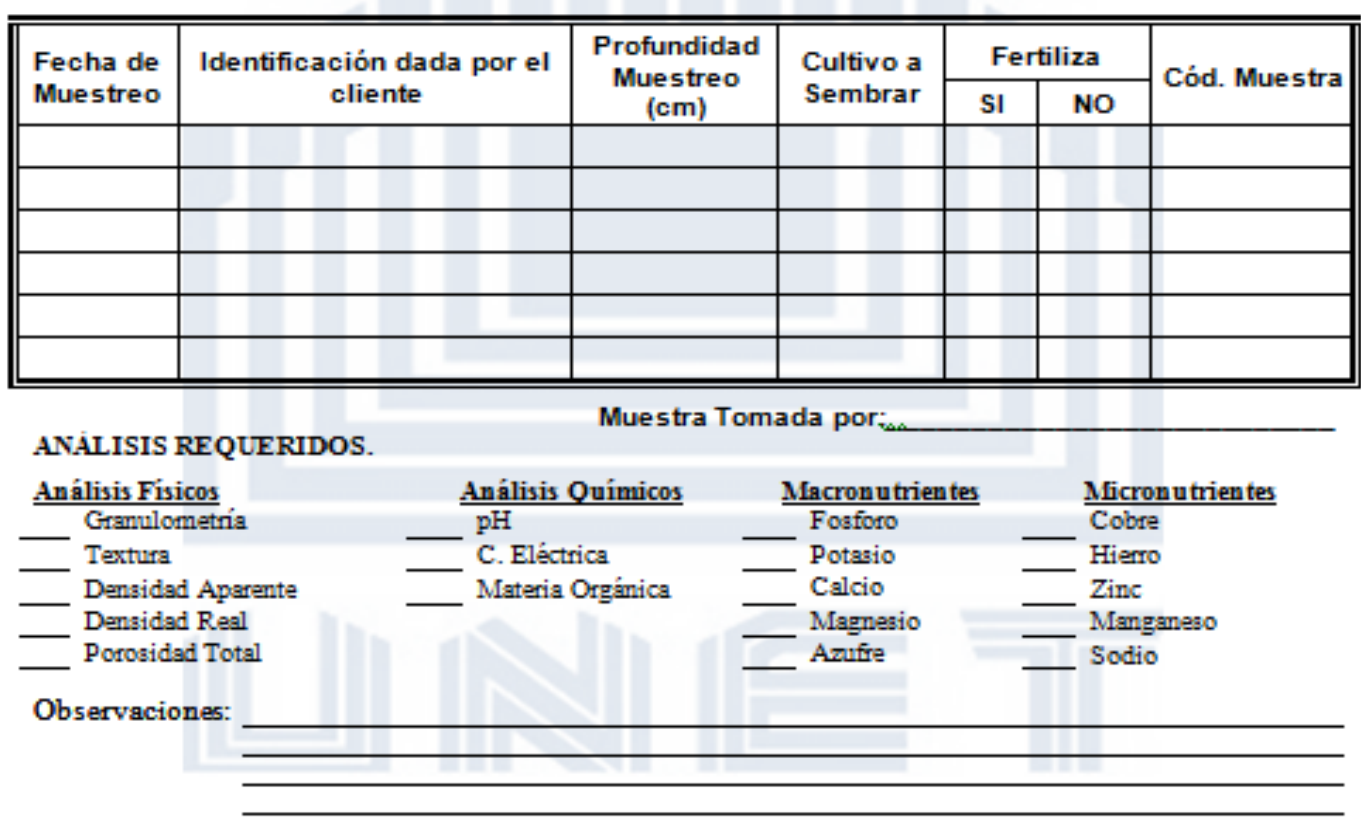

Figura 2. Planilla de recepción de datos de las muestras. 


\section{Resultados}

\subsection{Muestras de suelos del estado Táchira}

La localización de las muestras de suelos generó una capa vectorial (puntos) que permitió su representación cartográfica y a su vez, una base de datos con 644 registros (100\%) que fue exportado en formato dbf (database file) para ser abierto y procesado en excel. De dichos datos, 474 (73,6\%) están solo basados en la información existente en las planillas de recepción de análisis (municipio, parroquia y localidad, sin coordenadas geográficas o UTM); $132(20,5 \%)$ fueron corroborados con los usuarios de las fichas vía telefónica o e-mail, quienes suministraron la correcta ubicación de las muestras (envío de capturas de imágenes de Google Earth y Map con sus respectivas coordenadas); 14 (2,17\%) registros presentaron las coordenadas en las planillas; $2(0,31 \%)$ solo presentaron direcciones detalladas confiables y finalmente 7 (1,09\%) aunque no poseían planillas de recepción, fueron ubicadas a partir de la información digital existente (base de datos del Laboratorio). Para efectos del análisis se incluyeron 15 datos internos del Laboratorio (2,33\%), correspondientes a análisis realizados en los años 2006, 2009 y 2012 con el fin de incluir al menos cinco muestras en aquellos municipios que tenían bajo o nulo número de registros (como fue el caso de los municipios Simón Rodríguez, Francisco de Miranda y Sucre), para de esta forma completar la base de datos de los 29 municipios del estado Táchira (Figura 3).

En relación al registro de las muestras de los suelos durante el período 2015-2019 (Figura 4), se registraron 244 muestras (38,79\%) para el año 2015, 160 muestras (25,44\%) en 2016, 62 muestras (9,86\%) en 2017, 107 muestras (17,01\%) en 2018 y 56 muestras (8,9\%) en 2019, siendo 629 la totalidad de las muestras.

En el mismo orden de ideas, se obtuvo que el municipio con mayor cantidad de muestras de suelos analizadas es Fernández Feo con 115 (17,86\%), seguido por García de Hevía con 57 (8,85\%), Libertador con 51 (7,92\%), Michelena con 49 (7,61\%) y Lobatera con 36 (5,59\%). Por su parte, los municipios con menor número de muestras fueron San Judas Tadeo y Sucre con 6 muestras cada uno (0,93\%), Pedro María Ureña, Seboruco y Simón Rodríguez con 5 muestras cada uno (0,78\%) y Francisco de Miranda con 4 muestras (0,62\%) (Tabla 1). 
REVISTA DE LA UNIVERSIDAD DEL ZULIA. 3ㄹe época. Año 13 N 36, 2022 Luimar Álvarez et al. /// Gestión del Laboratorio Bioambiental de la Universidad Nacional Experimental... 173-188

DOI: http://dx.doi.org/10.46925//rdluz.36.12

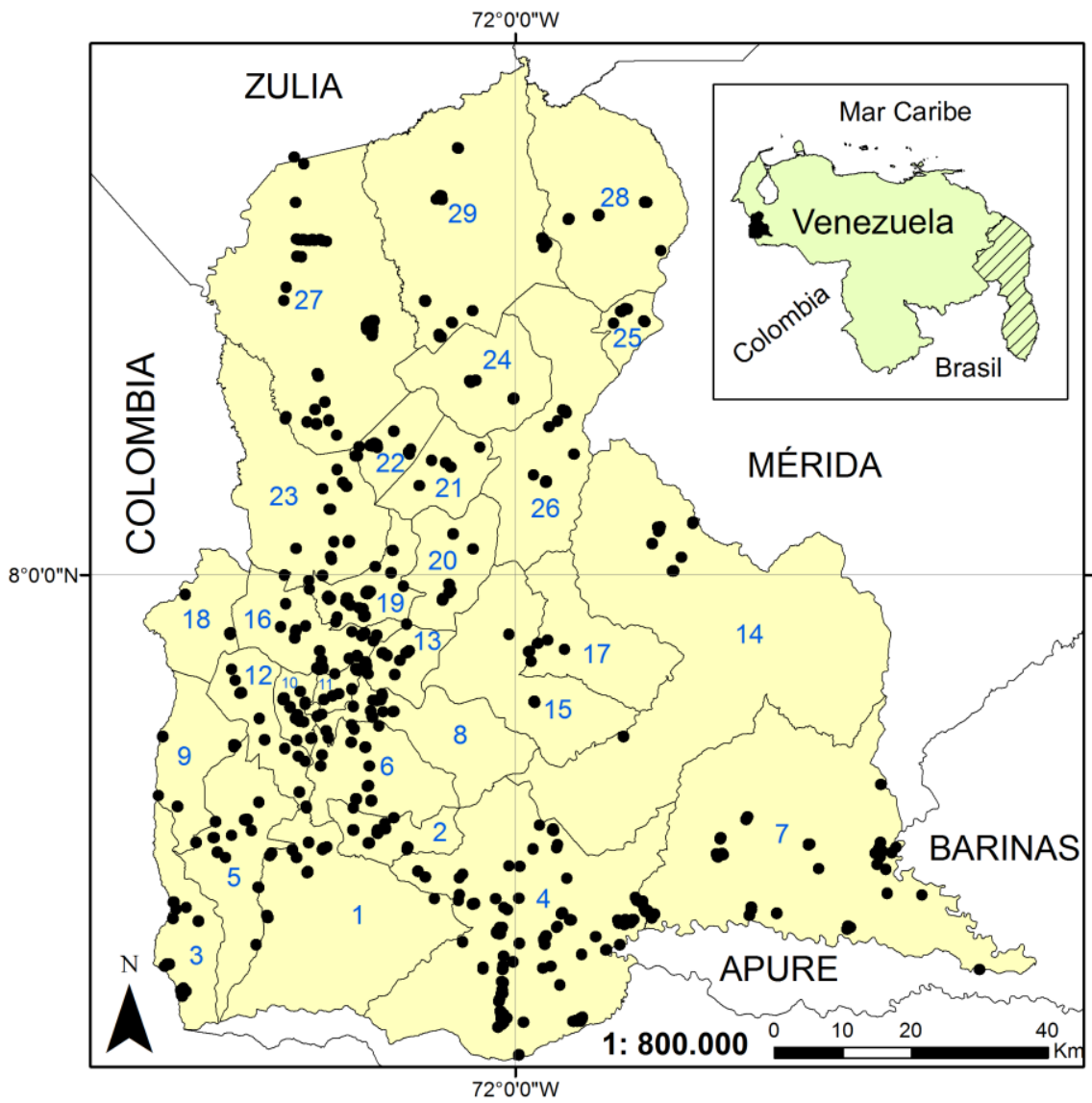

- Muestras de suelos Municipios

1, Cordoba

2, Torbes

3, Rafael Urdaneta

4, Fernandez Feo

5 , Junin

6, San Cristobal

7, Libertador

8, Cardenas

9, Bolivar

10, Independencia

11, Guasimos

12, Libertad

13, Andres Bello

14, Uribante

15 , Sucre

16, Lobatera

17, Francisco De Miranda

18, Pedro Maria Urena

19, Michelena

20 , Jose Maria Vargas

21, Seboruco

22, Antonio Romulo Costa

23, Ayacucho

24, San Judas Tadeo

25, Simon Rodriguez

26, Jauregui

27, Garcia De Hevia

28, Samuel Dario Maldonado

29, Panamericano

Figura 3. Ubicación de las muestras de suelo del estado Táchira entre los años 2015-2019 del Laboratorio Bioambiental UNET.

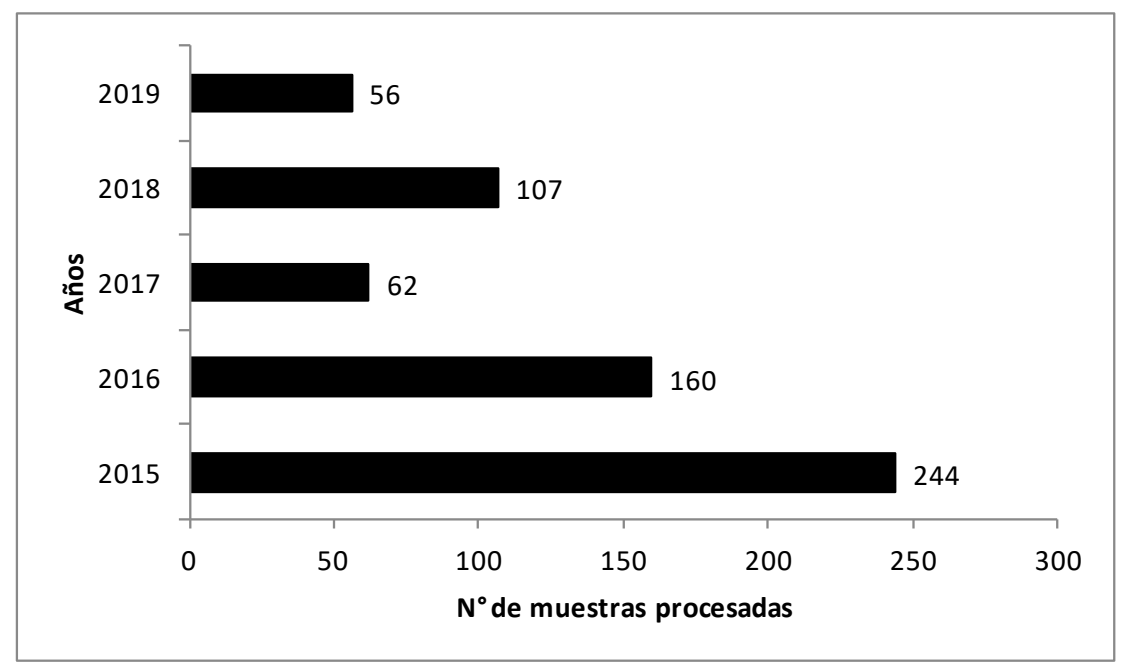

Figura 4. Número de muestras de suelos procesadas en el Laboratorio Bioambiental UNET en los años 2015-2019. 
Tabla l. Número de muestras analizadas por municipio durante el periódo estudiado (incluyendo las muestras de los años 2006, 2009 y 2012).

\begin{tabular}{lcc} 
Municipio & $\begin{array}{c}\text { № de } \\
\text { Muestras }\end{array}$ & $\%$ \\
\hline Fernández Feo & 115 & $17,86 \%$ \\
García de Hervia & 57 & $8,85 \%$ \\
Libertador & 51 & $7,92 \%$ \\
Michelena & 49 & $7,61 \%$ \\
Lobatera & 36 & $5,59 \%$ \\
Panamericano & 27 & $4,19 \%$ \\
San Cristobal & 24 & $3,73 \%$ \\
Cordoba & 23 & $3,57 \%$ \\
Independencia & 23 & $3,57 \%$ \\
Antonio Rómulo Costa & 21 & $3,26 \%$ \\
Ayacucho & 21 & $3,26 \%$ \\
Junín & 20 & $3,11 \%$ \\
Tórbes & 20 & $3,11 \%$ \\
Cárdenas & 19 & $2,95 \%$ \\
Rafael Urdaneta & 15 & $2,33 \%$ \\
Uribante & 15 & $2,33 \%$ \\
Andrés Bello & 13 & $2,02 \%$ \\
Libertad & 12 & $1,86 \%$ \\
Jauregui & 11 & $1,71 \%$ \\
José María Vargas & 11 & $1,71 \%$ \\
Samuel Dario Maldonado & 11 & $1,71 \%$ \\
Bolívar & 10 & $1,55 \%$ \\
Guásimos & 9 & $1,40 \%$ \\
San Judas Tadeo & 6 & $0,93 \%$ \\
Sucre & 6 & $0,93 \%$ \\
Pedro María Ureña & 5 & $0,78 \%$ \\
Seboruco & 5 & $0,78 \%$ \\
Simón Rodríguez & 5 & $0,78 \%$ \\
Francisco de Miranda & 4 & $0,62 \%$ \\
\hline Total & 644 & $100,00 \%$ \\
\hline
\end{tabular}

\subsection{Propiedades físico-químicas de los suelos del estado Táchira}

El análisis de los datos existentes permitió conocer los valores de referencia para los suelos del estado Táchira. En la Figura 5 se observa que más del 70\% de los suelos de los 
municipios del estado pertenecen a la clase textural franco arenoso $(\mathrm{Fa})$, un suelo de textura gruesa y el resto de suelos están caracterizados como franco arcillosos (FA), Franco Arcillo arenosos (FAa) y francos (F) que corresponden a suelos de textura media, resultados concordantes con los señalados en PALMAVEN (1992).

En el análisis estadístico descriptivo de los datos (Tabla 2), se observó que la mayor desviación estándar fue presentada en el Ca seguido del Mg, K y P respectivamente. Por otro lado, $\mathrm{MO}, \mathrm{pH}$ y $\mathrm{CE}$ fueron menores a una desviación. A partir de dichos valores, y según los criterios de Palmaven (1992), se consideró los valores de MO y P con una interpretación medio para los suelos del estado Táchira, en cuanto a los valores de K, Ca y Mg, la interpretación es alto. Por otra parte, el pH de los suelos del estado Táchira tienen la tendencia a ser fuertemente ácidos. Finalmente, en lo que respecta a la CE, esta es baja, es lo que indica que no afecta el desarrollo de los cultivos.

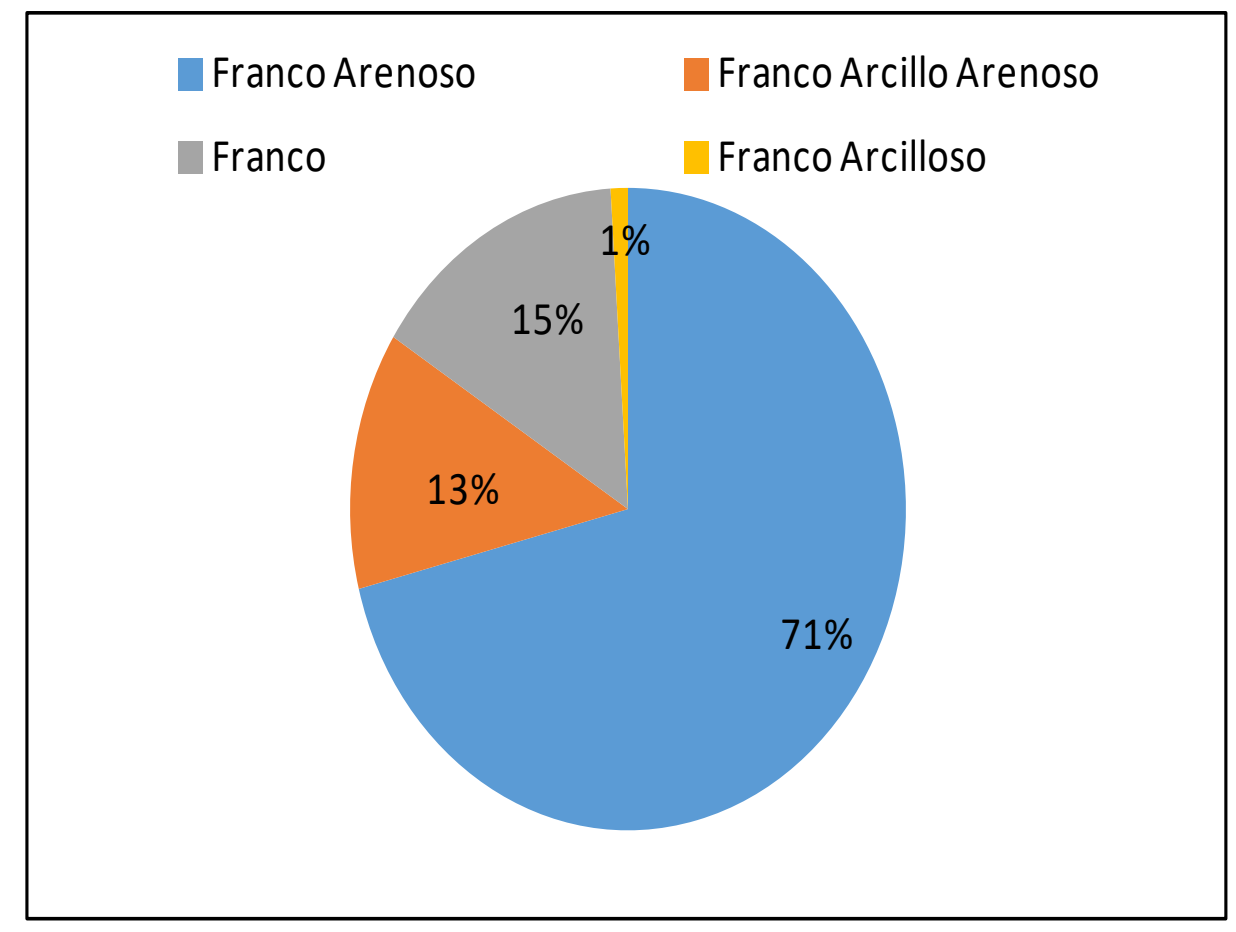

Figura 5. Porcentaje representativo de las clases texturales presentes en los datos analizados de suelos del estado Táchira 2015-2019. 
Tabla 2. Promedio general de los parámetros químicos determinados en los suelos del estado Táchira en el Laboratorio Bioambiental UNET 2015-2019.

\begin{tabular}{cccccc}
\hline Propiedad & Unidades & Media $\pm \mathrm{DE}$ & $\begin{array}{c}\text { Valores } \\
\text { mínimos }\end{array}$ & $\begin{array}{c}\text { Valores } \\
\text { máximos }\end{array}$ & Interpretación $^{*}$ \\
\hline $\mathrm{MO}^{\mathrm{a}}$ & $\%$ & $1,92 \pm 0,99$ & 0,02 & 7,86 & Medio \\
Pdisp & $\mathrm{mg} \cdot \mathrm{kg}^{-1}$ & $25 \pm 17$ & 2 & 242 & Medio \\
$\mathrm{K}^{\mathrm{c}}$ & $\mathrm{mg} \cdot \mathrm{kg}^{-1}$ & $155 \pm 99$ & 1 & 4188 & Alto \\
$\mathrm{Ca}^{\mathrm{c}}$ & $\mathrm{mg} \cdot \mathrm{kg}^{-1}$ & $894 \pm 500$ & 4,33 & 7435 & Alto \\
$\mathrm{Mg}^{\mathrm{c}}$ & $\mathrm{mg} \cdot \mathrm{kg}^{-1}$ & $176 \pm 134$ & 0,08 & 5369 & Alto \\
$\mathrm{pH}^{\mathrm{d}}$ & - & $4,95 \pm 0,39$ & 3,36 & 9,26 & $\begin{array}{c}\text { Fuertemente } \\
\text { ácido } \\
\mathrm{l}: 2,5\end{array}$ \\
$\mathrm{CE}$ & $\mathrm{mS} \cdot \mathrm{cm}^{\mathrm{e}}$ & $0,116 \pm 0,053$ & 0,01 & 1,25 & $\begin{array}{c}\text { Bajo: No afecta } \\
\text { ningún cultivo }\end{array}$ \\
$\mathrm{l}: 5$ & & & & &
\end{tabular}

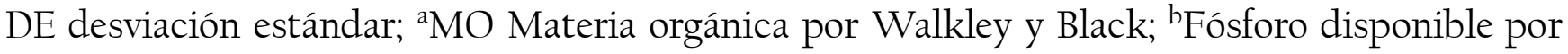
Bray I $\left(0,03 \mathrm{M} \mathrm{NH}_{4} \mathrm{~F}+0,025 \mathrm{M} \mathrm{HCl}\right) ;{ }^{~ c}$ Cationes intercambiables por extracción con acetato de amonio $1 \mathrm{M} \mathrm{pH} \mathrm{7,0;}{ }^{\mathrm{p}} \mathrm{pH}$ en agua 1:25 con $\mathrm{pH}$-metro; ${ }^{\circ}$ Conductividad eléctrica $1: 5$ con conductímetro. *Interpretación de acuerdo con el manual de análisis de suelo de PALMAVEN (1992).

En lo que respecta al análisis de correlación de Spearman entre las variables, se encontró que las mayores asociaciones positivas se dieron entre Pdisp y CE, seguido por Pdisp y $\mathrm{K}^{+}, \mathrm{Ca}^{2+}$ y $\mathrm{pH}$ principalmente, estas fueron seguidas $\mathrm{Mg}^{2+} \mathrm{y} \mathrm{pH}, \mathrm{K}^{+}$y $\mathrm{Ca}^{2+}, \mathrm{Ca}^{2+} \mathrm{y} \mathrm{Mg}^{2+}, \mathrm{K}^{+}$y Pdisp y finalmente por Pdisp y $\mathrm{Ca}^{2+}$. En cuanto a las restantes positivas aunque fueron mayores al estadistico de contraste, sus asociaciones fueron bajas. En último lugar, se obtuvieron relaciones negativas entre $\mathrm{MO}$ y Pdisp y entre Pdisp y $\mathrm{pH}$, siendo las únicas relaciones que se rechazan en la prueba de hipótesis (Tabla 3).

\section{Discusión}

Es notorio que el número de muestras procesadas por año ha tendido a disminuir debido principalmente a los incesantes paros universitarios en respuesta a la situación de crisis económica del país, que afectan directamente el continuo funcionamiento del Laboratorio Bioambiental de la UNET. Además, las continuas limitaciones de servicios públicos del estado Táchira, como la disponibilidad de combustible, que limita el traslado desde los distintos 
municipios hacia la ciudad San Cristóbal y viceversa, de las muestras tomadas por los agricultores, técnicos y público en general interesado en la realización de análisis de suelos.

Tabla 3. Coeficiente de correlación de Spearman entre las variables determinadas en el Laboratorio Bioambiental UNET.

\begin{tabular}{llllllll}
\hline & $\mathrm{MO}$ & Pdisp & $\mathrm{K}^{+}$ & $\mathrm{Ca}^{2+}$ & $\mathrm{Mg}^{2+}$ & $\mathrm{pH}$ & $\mathrm{CE}$ \\
\hline $\mathrm{MO}$ & 1 & & & & & & \\
Pdisp & $-0,037$ & 1 & & & & & \\
$\mathrm{~K}^{+}$ & 0,228 & $0,626^{*}$ & 1 & & & & \\
$\mathrm{Ca}^{2+}$ & 0,091 & $0,461^{*}$ & $0,580^{*}$ & 1 & & & \\
$\mathrm{Mg}^{2+}$ & 0,113 & 0,204 & 0,226 & $0,560^{*}$ & 1 & & \\
$\mathrm{pH}^{2}$ & 0,202 & $-0,026$ & 0,270 & $0,620^{*}$ & $0,573^{*}$ & 1 & \\
$\mathrm{CE}$ & 0,184 & $0,665^{*}$ & $0,514^{*}$ & 0,353 & 0,154 & 0,146 & 1 \\
\hline
\end{tabular}

Los valores marcados con * indican una correlación significativa $(\mathrm{p}<0,05)$. MO = materia orgánica. Pdisp = fósforo disponible. $\mathrm{CE}$ = conductividad eléctrica.

Se corroboró que el Laboratorio Bioambiental UNET maneja registros de los 29 municipios del estado Táchira (Tabla 1). Por esta razón se considera que existe una base de datos representativa de los suelos del estado, que permitió generar un análisis estadístico descriptivo (Tabla 2), que hasta el momento, no se habían determinado para nuestra entidad y que ahora pueden servir como referencia y estar a disposición de investigadores y público en general con interés en el recurso suelo. Sin embargo, en vista de los inconvenientes de la planilla de registro de muestras para ubicar las muestras, se consideró hacer modificaciones de la planilla, como la inclusión de un punto de referencia en la dirección y las coordenadas UTM, con el apoyo de Google Earth, Google map, entre otros, para las siguientes recepciones de muestras; recursos que no estaban disponibles para Martinez et al. (2006) al momento de geolocalizar la información de suelos tratada en el Laboratorio. 
Es pertinente acotar que los análisis de suelos presentan un nivel de nutrimentos bajo, medio, alto y muy alto, indicativo de la posibilidad de obtener una respuesta adicional con la aplicación de un fertilizante (mientras más alto el contenido del nutriente en el suelo, menor probabilidad de obtener una respuesta con la aplicación de fertilizantes) (PALMAVEN, 1992). En este sentido, Casanova (2005) ha señalado que la materia orgánica es altamente importante para el crecimiento de las plantas, ya que esta mediante la mineralización libera cantidades apreciables de nitrógeno, azufre y fósforo y algunos micronutrientes esenciales para el crecimiento y producción de las plantas. Asimismo, se considera que hay un contenido de $\mathrm{N}$ del $5,8 \%$ en la MO y que la MO mejora las características físicas y el almacén de energía para la vida microbiana del suelo (Trinidad-Santos y Velasco-Velasco, 2016). Por lo tanto, si un suelo posee poca materia orgánica es necesario incorporar antes de la siembra mediante la adición de abonos orgánicos y residuos vegetales e industriales apropiados. Para el caso del P disponible, de acuerdo con Bravo (2000) este representa apenas el 1\% del P total del suelo; por esta razón es recomendable en la mayoría de casos siempre aplicar fósforo, especialmente al momento de la siembra.

Por otro lado, los valores encontrados en las concentraciones de K, Ca y Mg (Tabla 1) son superiores en el caso del K y similares para el Ca y Mg en comparación con suelos de sabanas del estado Cojedes en Venezuela (Depablos et al., 2009); mientras que son superiores a los valores encontrados en suelos del estado Yaracuy (Borges et al., 2012). Asimismo, los valores encontrados para estos nutrimentos en el estado Táchira son superiores al nivel crítico tanto para el cultivo de hortalizas (FAO, 2013), como para forrajes (Morillo et al., 1989), lo que indica que los suelos del Táchira son aptos para el cultivo de estos rubros y para la actividad ganadera.

Con respecto al pH determinado, este corresponde a lo mencionado por Carrero et al. (2015), quienes consideran que en los Andes Venezolanos, la mayoría de los suelos tienden a ser ácidos. En estos suelos ácidos, el método de extracción de P disponible por Bray-Kurtz resulta ser más preciso que el método de Olsen (Carrero et al., 2015), tal como se realiza en el Laboratorio Bioambiental UNET. Los resultados de $\mathrm{pH}$ ácido y suelos desaturados son consecuentes con lo determinado por Ochoa et al. (2010) para suelos de los Andes venezolanos (estados Táchira, Mérida y Trujillo), además, es propio del trópico que los suelos presenten 
estas características debido a las altas precipitaciones y temperaturas (Lizcano et al., 2017). Los valores de $\mathrm{pH}$ indican que en la mayoría de los casos los productores deben realizar prácticas de corrección de este párametro mediante el encalado para mejorar la productividad de los cultivos (FAO, 2013).

De acuerdo con las correlaciones observadas (Tabla 3), se puede considerar que factores como el pH afectan la disponibilidad del Ca, así como también que la disponibilidad de este elemento está asociada a la disponibilidad de Mg, K y P. Igualmente, la disponibilidad de P y K tiene una relación directamente proporcional entre estos y la disponibilidad de ambos elementos está asociada a una mayor CE. Por el contrario, la MO no estuvo asociada a ninguno de los demás parámetros químicos determinados. En nuestros resultados, la correlación entre pH y Ca coincide con lo encontrado por Borges et al. (2012) para suelos del estado Yaracuy.

\section{Conclusiones}

De acuerdo a la cantidad de muestras y su ubicación, se considera que el Laboratorio Bioambiental UNET contiene datos representativos de los suelos espacialmente distribuidos del estado Táchira. Una alta proporción de estos tiene la condición textural gruesa, así como los valores promedio de los parámetros físico-químicos muestran que efectivamente los mismos son aptos para la actividad agrícola y ganadera. Lo anterior mencionado denota la posibilidad de obtener respuestas positivas a la aplicación de fertilizantes de acuerdo a la interpretación informada. Los resultados mostrados en este trabajo, son el punto de partida que permitirá posteriormente analizar y discutir por municipio, los parámetros físico-químicos de los suelos analizados, constituyendo así al Laboratorio como la referencia de suelos con potencial agrícola para la entidad.

\section{Agradecimientos}

Al personal técnico del Laboratorio Bioambiental de la UNET: Gloria Isabel Méndez, Ilse Marina Cárdenas, Aura Yanet Sánchez, Geisy Glendiurt Gámez, Marineisy Chacón, Yraima Colmenares, Francys Moros y Miguel Molina. Al personal Administrativo: Janet Rico, Lenis Cárdenas y Lisbeth Vela. Al Decanato de Extensión de la UNET y en especial a la Coordinación de Extensión Agraria. A la profesora Lorena Blanco de la UNET, por la corrección de estilo del 
presente manuscrito. Al personal académico, administrativo y obrero de la universidad que han colaborado y trabajado con el Laboratorio Bioambiental UNET desde su creación.

\section{Referencias}

Blanco, E. L., F. Rada, J. Paolini y J. A. Guerrero (2021). Effects of induced water deficit and biofertilization on growth dynamics and bulb yield of onion (Allium cepa L.) in a neotropical semi-arid environment. Canadian Journal of Soil Science, 101(3): 494-506. DOI: dx.doi.org/10.1139/cjss-2021-0011.

Borges, J. A, M. Barrios, E. Sandoval, Y. Bastardo y O. Márquez. (2012). Características físicoquímicas del suelo y su asociación con macroelementos en áreas destinadas a pastoreo en el estado Yaracuy. Bioagro, 24(2): 121-126.

Bravo, S. (2000). Aspectos básicos de química de suelos. Ediciones de la Universidad Ezequiel Zamora. Barinas, Venezuela. 249 p.

Caraballo, L. J. (2003). Caracterización económica de los municipios fronterizos del estado Táchira: Ayacucho, García de Hevia, Michelena y Lobatera. Aldea Mundo, 7(13), 46-61.

Carrero, A., A. Zambrano, E. Hernández, F. Contreras, D. Machado, G. Bianchi y R. Varela. (2015). Comparación de dos métodos de extracción de fósforo disponible en un suelo ácido. Avances en Química, 10(Especial), 29-33.

Casanova, E. (2005). Introducción a la ciencia del suelo. Consejo de Desarrollo Científico y Humanístico, 2da ed. Editorial Torimo. 482 p.

Castillo, L. D., M. A. Morffe, N. Albornoz y R. Mazuera (2014). El Táchira en cifras 2013. Vocación productiva, oferta de empleo y oferta educativa en el nivel superior en el estado Táchira. Observatorio Social del estado Táchira. Universidad Católica del Táchira. San Cristóbal, Venezuela. 309 p.

Depablos, L., S. Godoy, C. F. Chicco y J. Ordoñez. (2009). Nutrición mineral en sistemas ganaderos de las sabanas centrales de Venezuela. Zootecnia Tropical, 27(1), 25-37.

FAO (Organización de las Naciones Unidas para la Alimentación y la Agricultura). (2013). El manejo del suelo en la producción de hortalizas con buenas prácticas agrícolas. Consultado el 19 de octubre de 2021. https://www.fao.org/3/i336ls/i336ls.pdf

Lizcano, R., D. Olivera, D. Saavedra, L. Machado, E. R. Valencia, M. F. Moreno y M. F. Flórez. (2017). Muestreo de suelos, técnicas de laboratorio e interpretación de análisis de suelos. Servicio Nacional de Aprendizaje SENA. Bogotá, Colombia. 88 p. 
MARNR (Ministerio del Ambiente y de los Recursos Naturales Renovables). (1986). Atlas del estado Táchira.

Martínez, R., L. Chacón, J. González y H. Gómez. (2006). Aplicación de los SIG en la organización, análisis y divulgación de la información de suelo producida en laboratorio. Geoenseñanza, 11(1), 51- 62 .

Mondragón, M. (2014). Uso de la correlación de spearman en un estudio de intervención en fisioterapia. Movimiento Cientifico. 8 (1): 98-104.

Morillo, D., L. McDowell, C. Chicco, J. Perdomo, J. Conrad y F. Martin (1989). Nutritional status of beef cattle in specific regions of Venezuela. I. Macrominerals and forage organic constituents. Nutritional Report Int., 39: 1247-1262.

Ochoa, G., D Malagón, E. Palacios y J. Oballos (2010). Caracterización morfológica, química y mineralógica de suelos de la región andina venezolana. Revista Geográfica Venezolana, 51(1), 31-44.

PALMAVEN. (1992). Análisis de suelo y su interpretación. Serie Técnica. 2da ed. Ediciones PALMAVEN, S.A., filial de PDVSA. Caracas. 12 p.

Restrepo, L. y J. González. (2007). De Pearson a Spearman. Revista Colombiana de Ciencias Pecuarias. 20, 183-192.

Roberts, T.L. y J. L. Henry. (2001). El muestreo de suelos: los beneficios de un buen trabajo. Informaciones Agronómicas, 42, 4-7, 13.

Trinidad-Santos, A. y J. Velasco-Velasco. (2016). Importancia de la materia orgánica en el suelo. Agroproductividad, 9(8), 52-58.

Valero, M. A. (2009). Capítulo 55: Estado Táchira. En: GeoVenezuela, Fundación Empresas Polar (eds). Caracas, Venezuela. Pág. 128-241.

Villareal, J. E. (2018). Mapas de fertilidad como herramienta para zonificación de suelos en Panamá. Informaciones Agronómicas de Hispanoamérica, 31(diciembre), 32-39. 